\title{
CRITICAL EXPONENTS FOR A SEMILINEAR PARABOLIC EQUATION WITH VARIABLE REACTION.
}

\author{
R. FERREIRA, A. DE PABLO, M. PÉREZ-LLANOS AND J. D. ROSSI
}

\begin{abstract}
In this paper we study the blow-up phenomenon for nonnegative solutions to the following parabolic problem:

$$
u_{t}(x, t)=\Delta u(x, t)+(u(x, t))^{p(x)}, \quad \text { in } \Omega \times(0, T),
$$

where $0<p_{-}=\min p \leq p(x) \leq \max p=p_{+}$is a smooth bounded function. After discussing existence and uniqueness we characterize the critical exponents for this problem. We prove that there are solutions with blow-up in finite time if and only if $p_{+}>1$.

When $\Omega=\mathbb{R}^{N}$ we show that if $p_{-}>1+2 / N$ then there are global nontrivial solutions while if $1<p_{-} \leq p_{+} \leq 1+2 / N$ then all solutions to the problem blow up in finite time. Moreover, in case $p_{-}<1+2 / N<p_{+}$ there are functions $p(x)$ such that all solutions blow up in finite time and functions $p(x)$ such that the problem possesses global nontrivial solutions.

When $\Omega$ is a bounded domain we prove that there are functions $p(x)$ and domains $\Omega$ such that all solutions to the problem blow up in finite time. On the other hand, if $\Omega$ is small enough then the problem possesses global nontrivial solutions regardless the size of $p(x)$.
\end{abstract}

\section{INTRODUCTION}

We consider nonnegative solutions to the following parabolic semilinear problem with a reaction given by a variable exponent:

$$
\begin{cases}u_{t}(x, t)=\Delta u(x, t)+(u(x, t))^{p(x)}, & \text { in } \Omega \times(0, T), \\ u(x, 0)=u_{0}(x), & \text { in } \Omega,\end{cases}
$$

where $u_{0}(x)$ and $p(x)$ are two nonnegative continuous, bounded functions. For future references let us denote

$$
p_{-}=\inf _{x} p(x), \quad \text { and } \quad p_{+}=\sup _{x} p(x) .
$$

We study both cases $\Omega=\mathbb{R}^{N}$ or $\Omega$ a bounded smooth domain in which case we impose Dirichlet boundary conditions to our problem

$$
u(x, t)=0, \quad \text { on } \partial \Omega \times(0, T) .
$$

For this problem, we consider bounded initial data $u(x, 0)=u_{0}(x)$. Existence of a solution can be easily achieved, but uniqueness is subtle due to the

Key words and phrases. Blow-up, semilinear parabolic equations, critical exponents. 2000 Mathematics Subject Classification. 35B33, 35B40, 35K57. 
fact that $p(x)$ can be less than one in some region of $\Omega$. The difficulty comes from the non-Lipschitz character of the reaction, see [1]. Nevertheless, in this case we can prove existence of a maximal and a minimal solution. Moreover, a comparison principle among maximal solutions and among minimal solutions can be easily obtained. In the case $p_{-}<1$ we show that these solutions are always different for the initial value $u_{0} \equiv 0$ (and hence we have nonuniqueness). We are also able to prove that this is the only case of nonuniqueness, what is denoted by almost uniqueness in [16], when $p_{+}<1$ and $\Omega=\mathbb{R}^{N}$. We conjecture that almost uniqueness is true in the general case $p_{-}<1 \leq p_{+}$. When $p_{-} \geq 1$ uniqueness is standard. We will discuss these issues in the next section.

When dealing with a parabolic problem there are several interesting features to analyze, but the first step is to identify the so-called critical exponents. For $p$ constant we have that there are solutions to (1.1) with $T<\infty$ if and only if $p>1$. In this case, we have

$$
\lim _{t \nearrow T}\|u(\cdot, t)\|_{\infty}=+\infty,
$$

a phenomenon that is called blow-up in the literature and has deserved a great interest, see for instance the books [20], [21], the survey papers [2], $[3],[10],[14]$ and the references therein. However, up to our knowledge, this seems to be the first paper where the blow-up phenomenon is studied with a variable exponent as a reaction term.

Hence, the first critical exponent one has to look for in a parabolic problem is the blow-up exponent, a exponent such that there are solutions with blowup if and only if $p>p_{b}$. When $\Omega=\mathbb{R}^{N}$ and $p$ is constant we have $p_{b}=1$. Moreover, in this case there exists a second critical exponent, called Fujita exponent, see [7], [9], [11], [23] and the survey [14]. We quote also [5], [8], [12], [15], [17], [18], [19] and [22] for more references concerning Fujita exponents in other related problems. For $p>p_{f}=1+2 / N$ there are solutions with blow-up and global solutions while for $1<p \leq p_{f}$ every nontrivial solution blows up. Thus, the Fujita exponent separates regions of parameters for which all nontrivial solutions blow up and regions where there are both global and blow-up solutions.

In the Dirichlet case, we also have that the blow-up exponent is $p_{b}=1$ and there is no Fujita type exponent, since for $p>1$ there are always both global and blow-up solutions.

Our main aim in this paper is to find conditions on the variable exponent function $p(x)$, analogous to the above for constant $p$, in order to have existence or nonexistence of global solutions and/or blow-up solutions. These conditions are called blow-up conditions or Fujita type conditions.

We prove a sharp result concerning the blow-up occurence, Theorem 1.1, and two conditions of Fujita type in $\mathbb{R}^{N}$, Theorem 1.2, that are complemented with two examples. We also present a new phenomenon of Fujita type in bounded domains: roughly speaking, if $p(x)<1$ in some large set 
and $p(x)>1$ in some other set, also large, then every solution blows up, see Theorem 1.3.

First, let us look for the critical blow-up condition. In this case we have that $p_{+}=1$ is the critical condition for both (1.1) in $\mathbb{R}^{N}$ and in $\Omega$ bounded with (1.3). Indeed, it is enough for $p(x)$ to be larger than 1 in a small ball to have existence of blow-up solutions.

Theorem 1.1. For problem (1.1) in $\mathbb{R}^{N}$ or in a bounded domain with (1.3), we have:

(1) if $p_{+}>1$, then there are solutions that blow up in finite time.

(2) if $p_{+} \leq 1$, then every solution is global.

Next we look for the Fujita condition. For $\Omega=\mathbb{R}^{N}$ we have the following result that says that the value $p_{f}=1+2 / N$ plays a role. If $p(x)$ lies above $p_{f}$ everywhere then there are global solutions and if $p(x)$ lies below $p_{f}$ everywhere then every solution blows up, while there are functions $p(x)$ crossing the value $p_{f}$ that show that in this case we can have both situations.

\section{Theorem 1.2.}

(1) If $p_{-}>1+2 / N$, then problem (1.1) possesses global nontrivial solutions.

(2) If $1<p_{-}<p_{+} \leq 1+2 / N$, then all solutions to problem (1.1) blow up in finite time.

(3) If $p_{-}<1+2 / N<p_{+}$, then there are functions $p(x)$ such that problem (1.1) possesses global nontrivial solutions and functions $p(x)$ such that all solutions blow up.

In a bounded domain with Dirichlet boundary conditions we find the surprising fact that there is also a Fujita type phenomenon. In fact we can have that every nontrivial solution to (1.1) with Dirichlet boundary conditions (1.3) blows up. This has to be contrasted with the case $p$ constant in which there are always nontrivial global solutions. On the other hand if the domain is small enough then there are global solutions regardless the function $p(x)$. Both situations constitute the core of the following theorem.

\section{Theorem 1.3.}

(1) There are functions $p(x)$ and domains $\Omega$ such that all solutions to problem (1.1)-(1.3) blow up in finite time.

(2) If $\Omega \subset B_{r}\left(x_{0}\right)$ for some $x_{0} \in \mathbb{R}^{N}$ and $r<\sqrt{2 N}$ then problem (1.1)-(1.3) possesses global nontrivial solutions regardless the exponent $p(x)$.

(3) If $p_{-}>1$ then there are global solutions regardless the size of the domain $\Omega$.

The paper is structured as follows: in the following section we deal with the questions of existence, comparison and uniqueness for the solutions of our problems; in Section 3 we perform the study of the blow-up phenomenon. 


\section{EXISTENCE AND UNiQUENESS}

To begin our analysis, we discuss briefly existence and uniqueness of solutions to problem (1.1).

If $p_{-} \geq 1$, then the reaction term $f(x, u)=u^{p(x)}$ is continuous in both variables and locally Lipschitz in the second one. So, there exists a unique classical solution for any bounded initial datum, see [6]. Moreover, a comparison principle also holds: if $u_{0} \geq v_{0}$ (and in addition $u \geq v$ on $\partial \Omega$ in the Dirichlet problem case), then $u(x, t) \geq v(x, t)$. See [6] for $\Omega$ bounded and [1] for comparison in the whole space.

If $p_{-}<1$ we still have existence of a solution but uniqueness is not true in general. For instance, when $p(x)$ is constant $p(x) \equiv p<1$ and $\Omega=\mathbb{R}^{N}$, the function

$$
U(t)=c_{*} t^{\frac{1}{1-p}}, \quad c_{*}=(1-p)^{\frac{1}{1-p}},
$$

is a nontrivial solution with zero initial datum.

In the general case we can construct a maximal solution just by taking the limit

$$
\bar{u}=\lim _{\varepsilon \rightarrow 0} u^{(\varepsilon)},
$$

where $u^{(\varepsilon)}$ is the unique solution to our problem with initial condition $u^{(\varepsilon)}(x, 0)=u_{0}(x)+\varepsilon$, and with the reaction $f(x, t)=u(x, t)^{p(x)}$ replaced by

$$
f_{(\varepsilon)}(x, t)= \begin{cases}\left(u^{(\varepsilon)}(x, t)\right)^{p(x)} & \text { if } p(x) \geq \varepsilon \\ \varepsilon^{p(x)-1} u^{(\varepsilon)}(x, t) & \text { if } p(x)<\varepsilon .\end{cases}
$$

In the Dirichlet case we also replace the boundary condition by $u^{(\varepsilon)}=\varepsilon$ on $\partial \Omega$. Since the problem for $u^{(\varepsilon)}$ has comparison, we get in the limit a comparison result for maximal solutions. A minimal solution is obtained by taking limits for Lipschitz problems that approximate (1.1) from below. More precisely let

$$
\underline{u}=\lim _{\varepsilon \rightarrow 0} u_{(\varepsilon)},
$$

where $u_{(\varepsilon)}$ is the unique solution to the problem (1.1) with $f$ replaced by $f_{(\varepsilon)}$ and with the same initial data. Clearly we have, for any solution $u$ to problem (1.1), the inequalities

$$
0 \leq \underline{u} \leq u \leq \bar{u}
$$

Even more, any supersolution $z$ to the equation (1.1) satisfies $z \geq \underline{u}$, though comparison with $\bar{u}$ does not necessarily hold, but it does hold if $z$ is strictly positive, $z \geq \mu>0$. An analogous property is true for subsolutions. When $p(x)$ is constant, $p(x) \equiv p<1$ and $u_{0} \equiv 0$, we have that the minimal and maximal solutions are

$$
\underline{u}(x, t) \equiv 0, \quad \bar{u}(x, t)=U(t)=c_{*} t^{\frac{1}{1-p}},
$$

while a continuous family of different solutions between $\underline{u}$ and $\bar{u}$ exists, namely $u(x, t)=U(t-\tau)$ for $t>\tau>0, u(x, t)=0$ for $0 \leq \bar{t} \leq \tau$. 
We now prove that the same phenomenon occurs if only $p_{-}<1$ : a nontrivial solution exists when $u_{0} \equiv 0$. Therefore in this case $\underline{u} \neq \bar{u}$.

Theorem 2.1. Let $u_{0} \equiv 0$ in problem (1.1), and assume that the exponent satisfies $p(x) \leq \gamma<1$ for every $x \in D$, an open bounded subset of $\mathbb{R}^{N}$. Then the corresponding maximal solution satisfies $\bar{u}(x, t)>0$ for every $x \in D$, and for any $t>0$.

Proof. We construct a nontrivial positive subsolution. To this end let

$$
w(x, t)=a(t) \varphi_{1}(x),
$$

where $\varphi_{1}$ is the first eigenfunction of the laplacian with Dirichlet boundary condition in $D$, namely $\varphi_{1}$ satisfies $-\Delta \varphi_{1}=\lambda_{1} \varphi_{1}$ in $D, \varphi_{1}=0$ on $\partial D$, normalized according with $\max _{D} \varphi=1$. We want to choose $a(t)$ with $a(0)=$ 0 such that $w$ is a subsolution to (1.1). We first need, for $x \in D$,

$$
w_{t}-\Delta w=a^{\prime}(t) \varphi_{1}+\lambda_{1} a(t) \varphi_{1} \leq a(t)^{p(x)} \varphi_{1}^{p(x)}=w^{p(x)} .
$$

To get this inequality fulfilled it suffices to consider, for small $t$, for instance $t \leq 1$, the function

$$
a(t)=c t^{\frac{1}{1-\gamma}},
$$

with a suitable small constant $c>0$. Now, extending $w$ by zero outside $D$, we get that $w$ is a subsolution to (1.1), for $0 \leq t \leq 1$. This implies $\bar{u} \geq w>0$ in $D$, for $0 \leq t \leq 1$. Finally for times $k<t \leq k+1$ we compare with $w(x, t)$ replaced by $w(x, t-k)$.

In the case $p(x) \leq p_{+}<1$ for every $x \in \mathbb{R}^{N}$ we are able to prove almost uniqueness. We first need an universal lower estimate in the spirit of [1] and [16]. We recall that the proofs included in those papers work only when $p(x)$ is constant.

Lemma 2.2. Assume $p_{+}<1$ and let $u$ be any solution to problem (1.1) with initial datum $u_{0}(x) \not \equiv 0$. Then

$$
u(x, t) \geq c_{*} t^{\frac{1}{1-p_{+}}}, \quad c_{*}=\left(1-p_{+}\right)^{\frac{1}{1-p_{+}}},
$$

for every $x \in \mathbb{R}^{N}, 0 \leq t \leq 1$.

Proof. We want to refine the proof of Theorem 2.1. First of all, since comparison is not true for general solutions, we construct a strictly positive subsolution and perform a comparison argument in a set where our solution is also strictly positive.

It is clear that, since $u$ is a supersolution to the heat equation, we have that, given $x_{0} \in \mathbb{R}^{N}, r>0$ and $t_{0}>0$ small enough, then

$$
\mu=\min \left\{u(x, t): x \in B_{r}\left(x_{0}\right), t_{0} \leq t \leq t_{0}+1\right\}>0 .
$$

Now consider the function

$$
\widetilde{w}(x, t)=\mu+c t^{\alpha} \varphi_{1}\left(\frac{x-x_{0}}{r}\right), \quad \alpha=\frac{1}{1-p_{+}},
$$


where $\varphi_{1}$ is the first eigenfunction of the laplacian in the unit ball with $\varphi(0)=1$. We want to compare $u\left(x, t+t_{0}\right)$ with $\widetilde{w}(x, t)$ in $B_{r}(0) \times(0,1)$. The ingredients we need are the following:

- the problem has the comparison property: both functions satisfy $u(x, t+$ $\left.t_{0}\right) \geq \mu, \widetilde{w}(x, t) \geq \mu$, for $(x, t) \in B_{r}\left(x_{0}\right) \times(0,1)$;

- comparison of the initial conditions: $\widetilde{w}(x, 0)=\mu \leq u\left(x, t_{0}\right)$;

- comparison of the boundary data: $\widetilde{w}(x, t)=\mu \leq u(x, t)$ for $x \in \partial B_{r}\left(x_{0}\right)$;

- an inequality for the equation: substituting $\widetilde{w}$ in the equation we need $c>0$ to satisfy

$$
c \alpha t^{\alpha-1} \varphi_{1}+c t^{\alpha} \frac{\lambda_{1}}{r^{2}} \varphi_{1}-\left(c t^{\alpha} \varphi_{1}+\mu\right)^{p(x)} \leq 0 .
$$

This holds if we choose $c=\left(\alpha+\frac{\lambda_{1}}{r^{2}}\right)^{-\alpha}$.

This implies $u\left(x, t+t_{0}\right) \geq \widetilde{w}(x, t)$, and thus $u\left(x_{0}, t+t_{0}\right) \geq c t^{\alpha}+\mu$ for $0 \leq t \leq 1$. We finally let $t_{0} \rightarrow 0$ and $r \rightarrow \infty$ to get the desired result with the sharp constant.

With this estimate we now prove the almost uniqueness result.

Theorem 2.3. If $p_{+}<1$, then problem (1.1) in $\mathbb{R}^{N}$ has a unique solution for every nontrivial initial datum $u_{0} \not \equiv 0$.

Proof. Let $\underline{u}$ be the minimal solution to problem (1.1), and define for $k>1$ and $\eta>0$ fixed, the function $z(x, t)=k \underline{u}(x, t+\eta)$. We want to show that $z \geq \bar{u}$, the maximal solution, at least for small times. Letting $k \rightarrow 1$ and $\eta \rightarrow 0$ we conclude $\underline{u} \geq \bar{u}$, which means $\underline{u}=\bar{u}$.

Notice that since $p_{+}<1$ and $k>1$ the function $z$ is a supersolution to the equation. Therefore, by definition of maximal solution we only need to prove the comparison of the initial conditions $z(x, 0) \geq u_{0}(x)+\varepsilon$ for every $\varepsilon>0$ small enough, i.e.

$$
k u(x, \eta) \geq u_{0}(x)+\varepsilon .
$$

We thus consider the two following sets

$$
G=\left\{x \in \mathbb{R}^{N}: u_{0}(x) \leq \frac{1}{2} c_{*} \eta^{\alpha}\right\}, \quad B=\mathbb{R}^{N} \backslash G,
$$

and take $\varepsilon<\frac{1}{2} c_{*} \eta^{\alpha}$.

By the estimate (2.1), it suffices to prove that

$$
k c_{*} \eta^{\alpha} \geq u_{0}(x)+\varepsilon,
$$

which in the good set $G$ holds trivially. Let us then consider the set $B$. Since $u$ is a supersolution to the heat equation, by continuous dependence in time of the solution to this last equation, we have that for every $\delta<1$, $\delta \approx 1$, there exists $\eta>0$ small enough such that

$$
u(x, \eta) \geq \delta u_{0}(x) .
$$


Then (2.2) holds if

$$
(k \delta-1) \frac{1}{2} c_{*} \eta^{\alpha} \geq \varepsilon
$$

Taking $\delta^{-2}=k$, and $\varepsilon>0$ small to get the last inequality fulfilled, we obtain the desired comparison.

\section{BLOW-UP}

Now we focus our attention on the blow-up phenomenon, and consider the question of wether the solutions to our problems blow up or not. This leads to two types of results. Namely, on one hand, under which conditions on the reaction exponent $p(x)$ do we have existence of blow-up solutions or all solutions are globally defined. On the other hand, we also look for conditions on $p(x)$ such that every solution blows up or there exist also global solutions.

The first result concerning existence of blow-up solutions is an application of the Kaplan's method of eigenfunctions if $p(x)>1$ somewhere. We need the following version of Jensen's inequality. It uses some easy properties of the functional spaces $L^{p(x)}$, see for instance [4].

Lemma 3.1. Let $\mu$ be a positive measure in $B \subset \mathbb{R}^{N}$ with $\int_{B} d \mu=1$ and let $f \in L^{\gamma}(B, d \mu)$ and $1 \leq \delta \leq p(x) \leq \gamma$ for $x \in B$. Then, there exists a constant $c>0$ such that

$$
\int_{B}|f|^{p(x)} d \mu \geq c \min \left\{\left(\int_{B}|f| d \mu\right)^{\delta},\left(\int_{B}|f| d \mu\right)^{\gamma}\right\} .
$$

Proof. Following [4] we consider the space

$$
L^{p(x)}(B, d \mu)=\left\{g \text { measurable }: \int_{B}|g(x)|^{p(x)} d \mu<\infty\right\},
$$

with the norm

$$
\|g\|_{*} \equiv\|g\|_{L^{p(x)}(B, d \mu)}=\inf \left\{\tau>0: \int_{B}\left|\frac{g(x)}{\tau}\right|^{p(x)} d \mu \leq 1\right\} .
$$

The condition on $f$ guarantees $f \in L^{p(x)}(B, d \mu)$. It is easily verified that

$$
\int_{B}|f|^{p(x)} d \mu \geq \begin{cases}\|f\|_{*}^{\delta} & \text { if }\|f\|_{*} \geq 1 \\ \|f\|_{*}^{\gamma} & \text { if }\|f\|_{*} \leq 1\end{cases}
$$

On the other hand, a Hölder inequality holds in the above defined space, so we also have

$$
\int_{B}|f| d \mu \leq c_{1}\|f\|_{*}
$$

The constant $c_{1}$ is explicit in terms of the bounds on $p(x)$, and it satisfies $c_{1}<2$, see [4]. We also have used the fact that $\|1\|_{*}=1$. Therefore, we have proved the statement with $c=(1 / 2)^{\gamma}$.

Two useful consequences are given next. 
Corollary 3.2. In the above hypotheses we have

$$
\begin{aligned}
& \int_{B}|f|^{p(x)} d \mu \geq c \min \left\{\left(\int_{B}|f|^{\delta} d \mu\right),\left(\int_{B}|f|^{\delta} d \mu\right)^{\gamma / \delta}\right\}, \\
& \int_{B}|f| d \mu \geq 1 \Rightarrow \int_{B}|f|^{p(x)} d \mu \geq c\left(\int_{B}|f| d \mu\right)^{\delta} .
\end{aligned}
$$

We are now in a position to reproduce the classical Kaplan's argument for blow-up, see [13], and proceed with the proof of Theorem 1.1.

Theorem 3.3. If there exists some ball $B \subset \Omega$ in which the exponent function satisfies $p(x) \geq \delta>1$ then there exist solutions to problem (1.1) (with (1.3) in a bounded domain) that blow up in finite time.

On the other hand, if $p(x) \leq 1$ everywhere, then every nontrivial solution to problem (1.1) (with (1.3) in a bounded domain) is globally defined.

Proof. Take $\varphi$ the first eigenfunction of $-\Delta$ with Dirichlet boundary conditions in $B$ (with eigenvalue $\lambda$ ), normalized this time to have integral one. Let

$$
J(t)=\int_{B} u \varphi
$$

From the equation (1.1) and the above corollary we have,

$$
J^{\prime}=\int_{\Omega} u \Delta \varphi+\int_{\Omega} u^{p(x)} \varphi \geq-\lambda J+c J^{\delta},
$$

whenever $J(t) \geq 1$. Clearly this implies blow-up provided $J(0)$ is large. That is, if the initial datum is such that $J(0) \geq \max \left\{1,\left(2^{\delta} \lambda\right)^{1 /(\delta-1)}\right\}$, then the solution blows up.

To see that solutions are global when $p(x) \leq 1$ in the whole $\Omega$ it suffices to observe that the function

$$
w(t)=\left\|u_{0}\right\|_{\infty} e^{t}
$$

is a strictly positive supersolution to equation (1.1). Hence, for any $t_{0}>0$ the maximal solution to the problem is bounded in $\mathbb{R}^{N} \times\left[0, t_{0}\right]$ and then it is global. Observe that it is crucial that $w$ is strictly positive to use comparison arguments. Therefore, any solution is global.

We next consider the problem in the whole space $\mathbb{R}^{N}$ and study the socalled Fujita phenomenon.

Theorem 3.4. If $p_{-}>1+2 / N$, then problem (1.1) with $\Omega=\mathbb{R}^{N}$ possesses global solutions.

Proof. We only have to consider as supersolution a global solution to the problem with constant reaction exponent $p_{-}$such that it lies always below one, see [7].

Theorem 3.5. If $1<p_{-} \leq p_{+} \leq 1+2 / N$, then all solutions to problem (1.1) with $\Omega=\mathbb{R}^{N}$ blow up in finite time. 
Proof. Again the proof follows the classical methods of Fujita and Weissler for the constant exponent case, [7, 23], once we have established Jensen's inequality, Corollary 3.2. Assume then first that $1<p_{-} \leq p_{+}<1+2 / N$. Applying Kaplan's method with $\phi$ replaced by $\phi_{\mu}(x)=\mu^{N} \phi_{1}(\mu x)$, where $\phi_{1}$ is any nonnegative function satisfying $\int_{\mathbb{R}^{N}} \phi_{1}=1$ and $\Delta \phi_{1}+\phi_{1} \geq 0$ in $\mathbb{R}^{N}$ (for instance a gaussian), and using Lemma 3.1 we have

$$
J^{\prime} \geq-\mu^{2} J+c \min \left\{J^{p_{-}}, J^{p^{+}}\right\} .
$$

Now take $\mu>0$ small enough such that $J(0)>\left(\mu^{2} / c\right)^{1 /\left(p_{+}-1\right)}$. This is possible because of the restriction on $p_{+}$. In fact,

$$
\int_{\mathbb{R}^{N}} \mu^{N} \phi_{1}(\mu x) u_{0}(x) d x=J(0)>\left(\mu^{2} / c\right)^{1 /\left(p_{+}-1\right)}
$$

means

$$
\int_{\mathbb{R}^{N}} \phi_{1}(\mu x) u_{0}(x) d x>c^{-1 /\left(p_{+}-1\right)} \mu^{2 /\left(p_{+}-1\right)-N} \rightarrow 0 .
$$

Now, whenever $J(t)$ is small, (3.1) implies

$$
J^{\prime} \geq-\mu^{2} J+c J^{p^{+}}>\widetilde{c} J^{p^{+}} .
$$

This gives that $J(t)$ increases and for times $t>t_{1}$, where $t_{1}$ is such that $J\left(t_{1}\right)=1$, the inequality (3.1) becomes

$$
J^{\prime} \geq-\mu^{2} J+c J^{p^{-}}>\widetilde{c} J^{p^{-}} .
$$

This implies blow-up since $p_{-}>1$.

Let now $p_{+}=1+2 / N$. Here it suffices to show that the integral $\int_{\mathbb{R}^{N}} u(\cdot, t) d x$ is large for $t$ large. Without loss of generality we may assume, as in [23], that the initial datum is above some gaussian, $u_{0}(x) \geq G_{\varepsilon}(x)=$ $(4 \pi \varepsilon)^{-N / 2} \exp \left(-|x|^{2} / 4 \varepsilon\right)$. Therefore $u(x, t) \geq G_{t+\varepsilon}(x)$ for every $t \geq 0$. Now, by the integral representation of the solution of the semilinear equation, we have

$$
u(x, t) \geq \int_{0}^{t} G_{t-s} * u^{p(x)}(x, s) d s \geq \int_{0}^{t} G_{t-s} * G_{s+\varepsilon}^{p(x)}(x) d s .
$$

Integrating in $\mathbb{R}^{N}$ and using Corollary 3.2 we obtain

$$
\int_{\mathbb{R}^{N}} u(x, t) d x \geq \int_{0}^{t} \min \left\{\left(\int_{\mathbb{R}^{N}} G_{s+\varepsilon}^{p_{-}}(x) d x\right),\left(\int_{\mathbb{R}^{N}} G_{s+\varepsilon}^{p_{-}}(x) d x\right)^{p_{+} / p_{-}}\right\} d s .
$$

But a simple computation shows that

$$
\begin{aligned}
\int_{\mathbb{R}^{N}} G_{s+\varepsilon}^{p_{-}}(x) d x & =c \int_{\mathbb{R}^{N}}(s+\varepsilon)^{\left(1-p_{-}\right) N / 2} G_{(s+\varepsilon) / p_{-}}(x) d x \\
& =c(s+\varepsilon)^{\left(1-p_{-}\right) N / 2} .
\end{aligned}
$$

Since $p_{-} \leq 1+2 / N$ we have $\left(1-p_{-}\right) N / 2 \geq-1$, and thus the integral of this last term diverges as $t \rightarrow \infty$. 
Next we show some examples for the intermediate case, that is, for functions $p(x)$ with $1<p_{-}<1+2 / N<p_{+}$. In the first one every solution blows up and in the second one there are global solutions.

Example 1. Consider (1.1) in $\mathbb{R}$ and let $p(x)$ be any function such that $p(x)$ agrees with its minimum, that we fix between 1 and 2 , in the half line,

$$
p(x)=p_{-} \in(1,2) \quad \text { for } x \geq 0 .
$$

In this example we construct a subsolution with finite time blow-up. First, we note that the solution $u$ to (1.1) is positive for all $t>0$. Therefore we can take a nontrivial function $w_{0}(x) \leq u\left(x, t_{0}\right)$ with $w_{0}(0)=0$.

Now we consider the following problem

$$
\begin{cases}w_{t}=w_{x x}+w^{p_{-}}, & \text {in } \mathbb{R}^{+} \times(0, \tilde{T}), \\ w(0, t)=0, & \text { in }(0, \tilde{T}), \\ w(x, 0)=w_{0}(x), & \text { in } \mathbb{R}^{+} .\end{cases}
$$

It is clear from the above that $u$ is a supersolution to this problem. On the other hand, it is known that for this problem the blow-up and the Fujita exponents are given by $p_{b}=1$ and $p_{f}=2$ respectively, see [15].

Therefore any solution to our problem with the chosen reaction exponent blows up.

Example 2. We take,

$$
p(x)= \begin{cases}p_{+} & |x|>R \\ p_{-} & |x| \leq R\end{cases}
$$

where $p_{+}>N /(N-2)>1+2 / N$.

In this case, we construct a stationary supersolution. In the region $|x|>R$ we consider the explicit radial solution

$$
u(r)=c r^{-\alpha}, \quad \alpha=\frac{2}{p_{+}-1}, \quad c=(\alpha(N-2-\alpha))^{\frac{1}{p_{+}-1}} .
$$

In the inner region we consider a radial solution of

$$
\begin{cases}\Delta v+v^{p_{-}}=0, & x \in B_{R}(0) \\ v=c R^{-\alpha}, & x \in \partial B_{R}(0) .\end{cases}
$$

Notice that the function

$$
\bar{U}(x)= \begin{cases}v(|x|) & x \in B_{R}(0), \\ u(|x|) & x \in \mathbb{R}^{N} \backslash B_{R}(0),\end{cases}
$$

is a supersolution in the whole space if and only if $\left|v^{\prime}(R)\right| \leq\left|u^{\prime}(R)\right|$. In order to estimate $v^{\prime}(r)$ we consider the function

$$
w(r)=\frac{v(R r)}{c R^{-\alpha}},
$$

which verifies the problem

$$
\begin{cases}\Delta w+c^{p-1} R^{2+\alpha\left(1-p_{-}\right)} w^{p}=0, & x \in B_{1}(0), \\ w=1, & x \in \partial B_{1}(0) .\end{cases}
$$


Observe that for $R=0$ we obtain the constant solution $w(r)=1$. It is easy to check that for $R$ small enough we have

$$
w(r)=1+o(1) \quad \text { and } \quad w^{\prime}(r)=o(1) .
$$

This gives us $v^{\prime}(r)=o\left(R^{-\alpha-1}\right)$. On the other hand, $u$ verifies that $u^{\prime}(R)=$ $-c \alpha R^{-\alpha-1}$, then taking $R$ small enough, the function $\bar{U}$ is a supersolution to our problem.

Now we deal with the occurrence of a Fujita type phenomenon in a bounded domain. Actually we find sufficient conditions ensuring that every solution to problem (1.1)-(1.3) corresponding to a nontrivial nonnegative initial datum $u_{0}$, with $\Omega$ bounded, blows up. Note that this is an important difference with respect to the problem with a constant exponent in the reaction possed in a bounded domain. To build such examples we argue as follows: first we need a large region in which $p(x)$ lies below 1 (this will force the solution to grow in the whole $\Omega$ ) and a large region where $p(x)$ is above one (this is necessary for blow-up to occur, see Theorem 1.1).

We begin with a preliminary lemma.

Lemma 3.6. If there exists some ball $B_{R}\left(x_{0}\right) \subset \Omega$ in which the exponent function satisfies $0<\delta \leq p(x) \leq \gamma<1$, then any solution to problem (1.1)(1.3) verifies for every $x \in B_{R / 2}\left(x_{0}\right)$ that $u\left(x, t_{0}\right) \geq c R^{1 /(1-\gamma)}$, from some time $t_{0}>0$, with $c>0$ independent of $R$.

Proof. Without loss of generality let us suppose that the ball in the hypotheses is centered at the origin.

First, we analyze the easiest case, that is $p(x) \equiv \gamma<1$. It is clear that the maximal solution to problem

$$
\begin{cases}v_{t}=\Delta v+v^{\gamma}, & \text { in } B_{R}(0), \\ v=0, & \text { on } \partial B_{R}(0), \\ v=u_{0}, & \text { for } t=0,\end{cases}
$$

converges uniformly to a stationary solution, that we denote as $\bar{V}$. Indeed, we have a Lyapunov functional for (3.2),

$$
F(v)=\frac{1}{2} \int_{B_{R}(0)}|\nabla v|^{2}-\frac{1}{\gamma+1} \int_{B_{R}(0)} v^{\gamma+1} .
$$

It is also easy to check that the stationary solution $\bar{V}$ verifies that

$$
\bar{V}(x)=R^{2 /(1-\gamma)} \widetilde{V}(x / R),
$$

where $\widetilde{V}$ solves the stationary problem in the unit ball. Therefore, for any $x \in B_{R / 2}(0)$ it holds that

$$
\bar{V}(x)=R^{2 /(1-\gamma)} \tilde{V}(x / R) \geq R^{2 /(1-\gamma)} \min _{x \in B_{1 / 2}(0)} \tilde{V} .
$$


The proof of this case concludes taking into account that $u$ is supersolution to problem (3.2), therefore for $t_{0}$ large enough

$$
u(x, t) \geq \frac{1}{2} \bar{V}(x) \geq \frac{1}{2} R^{2 /(1-\gamma)} \min _{x \in B_{1 / 2}(0)} \widetilde{V},
$$

for all $t \geq t_{0}$ and $x \in B_{R / 2}(0)$.

For a general function $p$ satisfying $0<\delta \leq p(x) \leq \gamma<1$ we argue as before using comparison arguments which are valid for maximal solutions. We consider the maximal solution of the Dirichlet problem

$$
\begin{cases}v_{t}=\Delta v+v^{p(x)}, & \text { in } B_{R}(0), \\ v=0, & \text { on } \partial B_{R}(0), \\ v=v_{0}, & \text { for } t=0,\end{cases}
$$

which converges to a stationary solution $\bar{V}$ as $t \rightarrow \infty$. Observe that in this case the Lyapunov functional is given by

$$
F(v)=\frac{1}{2} \int_{B_{R}(0)}|\nabla v|^{2}-\int_{B_{R}(0)} \frac{|v|^{p(x)+1}}{p(x)+1} .
$$

Now, we pass to the unit ball with the change of scales,

$$
\bar{V}(x)=R^{\frac{1}{1-\gamma}} \tilde{V}(x / R),
$$

where $\widetilde{V}$ verifies

$$
\begin{cases}-\Delta \widetilde{V} \geq \widetilde{V}^{p(R x)}, & \text { in } B_{1}(0), \\ \widetilde{V}=0, & \text { on } \partial B_{1}(0) .\end{cases}
$$

In order to get rid of the dependence on $R$ of the exponent we observe that $\widetilde{V}$ is a supersolution of the problem

$$
\begin{cases}-\Delta z=z^{\delta} \chi_{\{z \geq 1\}}+z^{\gamma} \chi_{\{z<1\}} & \text { in } B_{1}(0), \\ z=0, & \text { on } \partial B_{1}(0),\end{cases}
$$

which does not depend on $R$. Summing up, we get that for $x \in B_{1 / 2}(0)$ and $t>t_{0}$

$$
u(x, t) \geq \frac{1}{2} \bar{V}(x)=\frac{1}{2} R^{\frac{1}{1-\gamma}} \widetilde{V}(x / R) \geq \frac{1}{2} R^{\frac{1}{1-\gamma}} \min _{x \in B_{1 / 2}(0)} z(x) .
$$

We are now ready to estate sufficient conditions ensuring blow-up occurrence for every solution to problem (1.1)-(1.3).

Theorem 3.7. For every $q$, an arbitrary function defined in the unit ball $B_{1}(0)$ verifying that $q(x)-1$ changes sign, there exists $L>0$ sufficiently large such that, if the ball $B_{L}\left(x_{0}\right) \subset \Omega$, then the solution to (1.1)-(1.3) blows up in finite time for any nontrivial nonnegative initial datum $u_{0}$, being $p(x) \equiv q\left(\left(x-x_{0}\right) / L\right)$, for every $x \in B_{L}\left(x_{0}\right)$. 
Proof. We assume for simplicity $x_{0}=0$. Since $L$ is large enough we have that $p$ is in the hypothesis of Lemma 3.6, that is, there exists a ball of radius $R_{1}$ large where $p$ satisfies $0<\delta \leq p(x) \leq \gamma<1$. Then, by this lemma we know that for some $t_{0}>0$ it holds that $u\left(x, t_{0}\right) \geq c R_{1}^{1 /(1-\gamma)}$, for any $x \in B_{R_{1} / 2}(0)$ (we assume again that this ball is centered at the origin).

It is not difficult to see that $u$ is supersolution to the following problem

$$
\begin{cases}\omega_{t}=\Delta \omega, & \text { in } \Omega \backslash B_{R_{1} / 2}(0), \\ \omega=0, & \text { on } \partial \Omega, \\ \omega=c R_{1}^{1 /(1-\gamma)}-1, & \text { on } \partial B_{R_{1} / 2}(0), \\ \omega(x, 0)=u\left(x, t_{0}\right), & \text { in } \Omega \backslash B_{R_{1} / 2}(0) .\end{cases}
$$

Thus $u(x, t) \geq \omega(x, t)$ for every $t>t_{0}$ and $x \in \Omega \backslash B_{R_{1} / 2}(0)$. Let us denote by $r(x)$ the stationary solution to the problem above. Since $\omega$ converges uniformly to $r$, it holds that $u(x, t) \geq r(x)-\delta$, for every $t>t_{1}$ for some $t_{1}>t_{0}$. On the other hand, we are assuming that the dilatation $L x$ is large enough. Thus there exists a ball $B_{2}$ of radius $R_{2}$ large where $p(x) \geq \delta>1$. Moreover, since

$$
J\left(t_{1}\right)=\int_{B_{2}} u \varphi_{B_{2}} \geq \int_{B_{2}}(r(x)-\delta) \varphi_{B_{2}}>2,
$$

by Theorem 3.3 we conclude that the solution blows up in finite time.

Now we prove that when $\Omega$ is contained in a small ball then there are global solutions regardless the size of $p(x)$.

Theorem 3.8. If there exists some ball $B_{r}\left(x_{0}\right) \supset \Omega$ with $r<\sqrt{2 N}$, then there are global solutions to (1.1) with Dirichlet boundary conditions (1.3) for every $p(x) \geq 0$.

Proof. We only note that the function

$$
w(x)=\frac{2 N-\left|x-x_{0}\right|^{2}}{2 N}
$$

is a supersolution of (1.1). Indeed, since $r<\sqrt{2 N}$ we have that $w(x)>0$ at $\partial \Omega$. Moreover, $w(x) \leq 1$, hence $w(x)^{p(x)} \leq 1=-\Delta w(x)$.

Theorem 3.9. Let $p_{-}>1$, then there is a global nontrivial solution to (1.1)-(1.3)

Proof. Let

$$
z(x, t)=\varepsilon e^{-\lambda t} \varphi_{1}(x),
$$

where $\varphi_{1}$ is the first eigenfunction of the Laplacian in $\Omega$ with Dirichlet boundary conditions, normalizad with $\max _{x} \varphi_{1}(x)=1$.

Then we have that $z$ is a supersolution provided $\lambda$ and $\varepsilon$ are small. In fact, we have

$$
z_{t}(x, t)=-\lambda z(x, t)
$$


and

$$
\begin{aligned}
\Delta z(x, t)+z^{p(x)}(x, t) & =-\lambda_{1} z(x, t)+\left(\varepsilon e^{-\lambda t} \varphi_{1}(x)\right)^{p(x)} \\
& \leq-\lambda_{1} z(x, t)+\left(\varepsilon e^{-\lambda t} \varphi_{1}(x)\right)^{p_{-}} .
\end{aligned}
$$

And hence it suffices with

$$
\lambda z(x, t) \leq \lambda_{1} z(x, t)-z^{p_{-}}(x, t),
$$

that is,

$$
\left(\lambda_{1}-\lambda\right) z(x, t) \geq z^{p_{-}}(x, t),
$$

which holds choosing $\lambda$ and $\varepsilon$ small enough, since $p_{-}>1$.

\section{Acknowledgements.}

RF partially supported by grants CCG07-UCM/ESP-2393 UCM-Com. de Madrid and MTM2005-08760-C02-01, MEC Spain.

AdP and MP partially supported by grant MTM2005-08760-C02-02, MEC Spain.

JDR partially supported by project MTM2004-02223, MEC, Spain, and by UBA X066 and CONICET, Argentina.

\section{REFERENCES}

[1] J. Aguirre and M. Escobedo. A Cauchy problem for $u_{t}-\Delta u=u^{p}$ with $0<p<1$. Asymptotic behaviour of solutions. Ann. Fac. Sci. Toulouse 8 (1987), 175-203.

[2] C. Bandle and H. Brunner. Blow-up in diffusion equations: a survey. J. Comp. Appl. Math. 97 (1998), 3-22.

[3] K. Deng and H. A. Levine. The role of critical exponents in blow-up theorems: The sequel. J. Math. Anal. Appl. 243 (2000), 85-126.

[4] X. Fan and D. Zhao. On the spaces $L^{p(x)(\Omega)}$ and $W^{m, p(x)}(\Omega)$. J. Math. Anal. Appl. 263 (2001), 424-446.

[5] R. Ferreira, A. de Pablo and J. L. Vazquez. Classification of blow-up with nonlinear diffusion and localized reaction. J. Differential Equations, 231 (2006), 195211.

[6] A. Friedman. Partial Dofferential Equation of Parabolic Type. Prentice-Hall, Englewood Cliffs, 1969.

[7] H. Fujita. On the blowing up of solutions of the Cauchy problem for $u_{t}=\Delta u+$ $u^{1+\alpha}$. J. Fac. Sci. Univ. Tokyo Sect. I. 13 (1966), 109-124.

[8] V.A. Galaktionov and H.A. Levine. On critical Fujita exponents for heat equations with nonlinear flux conditions on the boundary, Israel J. Math. 94 (1996), 125146.

[9] V.A. Galaktionov and H.A. Levine. A general approach to critical Fujita exponents in nonlinear parabolic problems, Nonlinear Anal. 34 (1998), 1005-1027.

[10] V. Galaktionov and J. L. Vázquez. The problem of blow-up in nonlinear parabolic equations, Discrete Contin. Dynam. Systems A 8 (2002), 399-433.

[11] K. Hayakawa. On the nonexistence of global solutions of some semilinear parabolic equations. Proc. Japan Acad. 49 (1974), 503-525.

[12] W. Huang, J. Yin and Y. Wang. On critical Fujita exponents for the porous medium equation with a nonlinear boundary condition. J. Math. Anal. Appl., 286 (2003), 369-377. 
[13] S. Kaplan. On the growth of solutions of quasilinear parabolic equations. Comm. Pure Appl. Math. 16 (1963), 305-330.

[14] H. A. Levine. The role of critical exponents in blowup theorems. SIAM Rev. 32 (1990), 262-288.

[15] N. Mizoguchi and E. Yanagida. Critical exponents for the blowup of solutions with sign changes in a semilinear parabolic equation. II. J. Differential Equations 145 (1998), 295-331.

[16] A. de Pablo and J. L. Vazquez. Balance between strong reaction and slow diffusion. Comm. Partial Diff. Eq. 15 (1990), 159-183.

[17] Y.W. Qi. The critical exponents of parabolic equations and blow-up in $R^{n}$. Proc. Roy. Soc. Edinburgh Sect. A 128 (1998), 123-136.

[18] Y.W. Qi and H.A. Levine. The critical exponent of degenerate parabolic systems, Z. Angew. Math. Phys. 44 (1993), 249-265.

[19] F. Quirós and J.D. Rossi. Blow-up sets and Fujita type curves for a degenerate parabolic system with nonlinear boundary conditions, Indiana Univ. Math. J. 50 (2001), 629-654.

[20] P. Quittner and P. Souplet. Superlinear parabolic problems. Blow-up, global existence and steady states. Birkhäuser Verlag, Basel, 2007.

[21] A. Samarski, V. A. Galaktionov, S. P. Kurdyumov and A. P. Mikailov. Blow-up in quasilinear parabolic equations. Walter de Gruyter, Berlin, 1995.

[22] C. Wang and S. Zheng. Critical Fujita exponents of degenerate and singular parabolic equations. Proc. Roy. Soc. Edinburgh Sect. A. 136 (2006), 415-430.

[23] F. B. Weissler. Existence and nonexistence of global solutions for a semilinear heat equation. Israel J. Math. 38 (1981), 29-40.

RAÚL FERREIRA

Departamento de Matemáticas,

U. COMPlutense DE Madrid,

28040 MADRID, SPAIN.

E-MAIL: raul_ferreira@ucm.es

Arturo de Pablo

Departamento de Matemáticas,

U. CARlos III de Madrid,

28911 Leganés, SPAin.

E-MAIL: arturop@math.uc3m.es

Mayte PÉRez-LLanos

Departamento de Matemáticas,

U. CARLOS III DE MADRID,

28911 LEGANÉS, SPAIN.

E-MAIL: mtperez@math.uc3m.es

Julio D. Rossi

IMDEA MATEMÁticas, C-IX, CAMPUS UAM,

MADRID, SPAIN

On leave from Departamento de Matemática, FCEyN UBA (1428) Buenos Aires, Argentina.

jrossi@dm.uba.ar 